Cahiers de recherches médiévales

Journal of medieval studies

2 | 1996

Regards sur le Moyen Âge

\title{
Bertran de Born, itinéraires européens
}

\section{Martine Dauzier}

\section{OpenEdition}

\section{Journals}

Édition électronique

URL : https://journals.openedition.org/crm/2504

DOI : $10.4000 / \mathrm{crm} .2504$

ISSN : $1955-2424$

Éditeur

Honoré Champion

Édition imprimée

Date de publication : 12 décembre 1996

Pagination : 179-186

ISSN : 1272-9752

\section{Référence électronique}

Martine Dauzier, «Bertran de Born, itinéraires européens », Cahiers de recherches médiévales [En ligne],

2| 1996, mis en ligne le 04 février 2008, consulté le 15 décembre 2022. URL : http://

journals.openedition.org/crm/2504; DOI : https://doi.org/10.4000/crm.2504 


\section{Bertran de Born, itinéraires européens}

Comment pour un écrivain échapper au purgatoire de l'oubli? En passant par l'Enfer, répondrait-on plaisamment à propos de Bertran de Born, troubadour périgourdin de la fin du XII' siècle, tel qu'en lui-même la littérature européenne le change. En effet, si les troubadours ont eu du mal à réapparaître dans la culture française, sans doute fût-ce moins long dans certains pays d'Europe pour certains poètes qui échappent à la lecture savante, éveillant des images puis des légendes qui amènent à des réincarnations que les spécialistes n'apprécient guère' ${ }^{1}$. Rares sont les troubadours aussi souvent rappelés à la vie que celui-ci, sinon le Jaufré Rudel de La Princesse lointaine ${ }^{2}$; car rares les poètes: en France, pour un Villon, un Rimbaud, combien d'autres personnages littéraires recrées? Quelles sont les conditions et les modalités de ces successives réincarnations d'un troubadour? Comment les vicissitudes d'une histoire complexe, pour ne pas dire confuse, de conflits familiaux, féodaux et royaux entre 1180 et 1200 , peuvent-elles accompagner le temps qui passe? Nous verrons qu'il s'agit de faire revivre plus que tout un poète, aimé des poètes, et un poète de l'énergie à l'ardeur capable d'animer les drames.

\section{Points d'ancrage littéraires pour une aventure héroïque}

Autant les poèmes de Bertran de Born parlent des rois, et conseillent les rois, ceux d'Angleterre, de France et d'Aragon, autant l'histoire des rois reste silencieuse à son sujet. A peine quelques chartes de l'abbaye voisine de son château de Hautefort nous éclairent-elles, entre 1179 et 1215 , la vie familiale, féodale, et religieuse d'un petit châtelain périgourdin à une époque particulièrement mouvementée, celle des luttes qui opposent les Plantagenêts et le roi de France, celle aussi des conflits entre les Plantagenêts, père et fils, frères entre eux. La chronique est avare de notations, même en pays d'oc.

Réalité documentaire fragmentaire sans doute, mais ce chevalier - le miles des chartes a laissé dans les chansonniers occitans quarante sirventes, cansos, planh d'attribution sûre, dont certains copiés dans plus de dix chansonniers, preuve s'il en est de leur succès ${ }^{3}$.

Poète, ami des poètes comme Folquet de Marseille, Guilhem de Berguedan et Conon de Béthune, cet auteur de sirventes a créé un modèle poétique pour rendre compte de l'actualité qui n'apparaissait auparavant dans les vers ou les sirventes moraux que sous la forme de quelques vers allusifs, ou d'une cobla. A l'origine de véritables genres, il façonne les formes

\footnotetext{
${ }^{1}$ Voir Imntraud Mörgner, Vie et aventures de la Trobainitz Béatrice d'après les témoignages de sa ménestrelle Laura, Darmstadt, 1974 ( traduction française 1983), où la trobainizz est confrontée au féminisme, à l'écologie et au réalisme socialiste par la romancière allemande.

${ }^{2}$ A. Döblinconsacre un chapitre de son demier roman (1945-1946) Hamlet oder die lange Nacht nimmt ein Ende à la princesse de Tripoli, qui se nourrit de jeunes chiots et épuise ses amants, et à un bien trompeur « amour de loin».

${ }^{3}$ Voir l'édition de Gérard Gouiran, L'amour et la guerre - l'auvre de Bertran de Born, Aix-enProvence, 1985.
} 
de l'enueg, l'escondig et du plazer, inspirant Peire de Bergerac, Peire Cardenal ou des auteurs catalans. Il ouvre le sirventes sur la geste grâce en particulier à la poésie des toponymes et des noms illustres. Il multiplie plus qu'aucun autre ce style proprement épique, celui des listes évoquant des châtellenies, buttes fortifiées, villes franches, des alliés et des ennemis. Menaces, souhaits, regrets renvoient aux topoi de la vantardise par une poésie de l'hyperbole et du contraste. Le « je », et ses batailleuses vertus, s'affirme omniprésent dans une société de l'abondance de bruits et de couleurs, où la guerre est d'abord une immense fête de la destruction, une danse dans le tournoiement des combats et des assauts, alors que la Nature, comme dans les chansons courtoises, renouvelle feuilles et fleurs.

Mais à une époque où même les chroniqueurs répugnent au strictement circonstanciel, lui préférant le monument, comment sauver pour la postérité les fugaces inscriptions de l'actualité ? Le poète compte sur la vigueur de son style et le jongleur de la génération qui suit adopte, au XII' siècle, une stratégie de l'explication précédant l'interprétation chantée. De courtes biographies ñ deux vidas pour Bertran de Born - permettent de situer l'auteur des chants tandis que les razos rendent « raison », glosent les poèmes. Sur 350 troubadours, 46 voient leurs poèmes commentés en 113 razos ; or Bertran de Born vient de très loin en tête avec 19 de ces petits récits. Si certains ne sont qu'une mise en prose du poème, éclaircissant ou non quelques allusions, d'autres centrent l'intérêt sur la personne de Bertran, parfait chevalier guerroyeur et parfait compagnon, impressionnant de passion farouche et de fidélité, figure symétrique du fin amic des cansos. Ces textes possèdent souvent une construction narrative très ferme, telle la razo du face-à-face orgueilleux du chevalier vaincu, refusant de trahir son ami le Jeune Roi, avec le vieux roi Henri II. Vidas et razos confondent métaphores et réalités historiques, ce que les médiévistes français, de Jeanroy à Boutière, leur reprocheront vivement, mais créent une trame narrative persuasive à force de répétitions et d'auto-références.

Poèmes et surtout razos fournissent autant qu'une matière une «conjointure » où peut s'ancrer la mémoire. On saisit l'organisation d'un modèle hérö̈que avec une situation relativement obscure de départ, vécue dans les interstices et les silences de la grande Histoire, celle d'un petit châtelain, à peine co-seigneur. Or, mis soudain en présence de tous les grands rois d'Occident, le voilà célébré pour ses exploits de conseiller, guerrier, poète et prophète, mais vite guetté par des échecs retentissants : châtelain perdant son château, vassal perdant son roi, ami ses compagnons. Autant d'épreuves que parachève une disparition dans le silence du cloître.

\section{Des alliés substantiels en Europe}

Pour échapper à la catastrophe occitane, à la disparition violente d'une société et d'une langue, donc de toute une culture, le troubadour comptera sur des « alliés substantiels », pour beaucoup européens plus que français ou occitans. Cela ne saurait nous surprendre à propos de ces voyageurs ou de ces exilés, de ces créateurs polyglottes tel un Raimbaut de Vaqueyras, de ces hommes qui eurent pour interprètes des jongleurs comme Cercamon, «Court le monde » et pour copistes des scribes de Catalogne ou d'Italie.

Leurs poèmes résonnèrent à Florence et Dante, en consacrant toute la fin du Chant XXVIII de L'Enfer à l'apparition terrible de Bertran de Bom au fond du neuvième fossé du 
huitième cercle, parmi les semeurs de discorde, aux côtés de Mahomet et de Malatesta, fait sortir à jamais du gouffre de silence cette ombre mise en pièces, ce buste décapité, cette tête qui parle et n'en finira pas d'inspirer les illustrateurs de La Divine Comédie, de Botticelli à Blake, de Doré à Dali. Combien de lecteurs des troubadours jusqu'à nos jours rencontrent d'abord ceux que l'on a fini par appeler «les troubadours de Dante», Bertran, de Bom, Sordel et Folquet de Marseille, Ezra Pound en étant pour notre siècle le plus fameux exemple. Cette gloire a son revers : pour les Italiens, parfois Dante suffit, sans qu'on se croit obligé de relire les poèmes! Le livret demandé à Filiberto Balegno par Ponchielli- le créateur de La Gioconda ñ pour l'opéra Bertrando del Bomio, qui aurait dû être créé en 1858, à Turin, et qui ne le fut jamais ${ }^{4}$, en est la preuve. Balegno emprunte à Dante l'image du diviseur de familles puisque Henri II est trahi et combattu par ses fils, à l'instigation d'un grand seigneur aquitain, Bertran; jaloux et furieux, celui-ci pousse celle qu'il aime en vain au suicide par le poison. Le méchant se condamne par là-même à l'Enfer. Rien du poète, sinon le nom et sa trop noire légende.

Malgré ses liens avec l'histoire des Plantagenêts, le XIX siècle anglais lui préfère, chez le poète Swinburne, Rudel et des princesse de Tripoli aux charmes préraphaélites, ou des romans historiques exaltant, à la Walter Scott, un Richard Coeur de Lion de légende. En 1900 un auteur à succès, Sir M. Hewlett, fait cependant intervenir le poète auprès de Richard mais pour mieux l'insulter, le rendre laid et exécrable, le condamner comme un démon mais démon de petite volée, plus mesquin que belliqueux, ridicule «chien galeux ». Le texte sera traduit en français en 1950 à Charleroi .

Les Espagnols ont leur Amadis, leur Cid Campeador, sans avoir à glaner ailleurs le pittoresque méridional! Chez les Catalans, l'autonomiste et trobaïre Victor Balaguer fait au troubadour une place parmi les grands entraîneurs de peuples, Pierre l'Ermitte et Rouget de l'Isle. Les échos des sirventes résonnent de siècle en siècle, de symbole à symbole, unissant profane et sacré. Mais il n'écrivit plusieurs drames en catalan que sur la Croisade des Albigeois qui, il est vrai, scella une part du destin de la Catalogne.

Tout change de l'autre côté du Rhin comme le signale déjà en 1810 Germaine de Staël dans De l'Allemagne ${ }^{6}$. La réflexion qu'avait commencée le XVW' siècle, y compris parisien ou provençal, sur les liens entre les cultures méridionales et la poésie avant de l'oublier dans la prédilection révolutionnaire pour l'Antiquité, est enrichie par l'école allemande. Les philologues sont attirés dès l'abord par la figure du poète politique; Walter von der Vogelweide ne fait-il pas partie de leur panthéon poético-national ? En France, ce sont plutôt les cansos qui retiennent Rochegude et les premiers volumes de Raynouard.

La réflexion sur l'origine des poésies nationales entreprise par A.W. Schlegel (1818) culmine avec les travaux de Fr. Diez en 1824 dont les traductions - où se note la prédilections pour les pièces historiques - seront reprises par plusieurs générations. L'interaction entre philologie et création sera à partir des Romantiques une spécificité allemande. Uhland, professeur de littérature allemande à Tübingen, auteur d'un ouvrage sur Walter von der Vogelweide, dramatise dans une ballade de 1831 l'affrontement inventé par

\footnotetext{
${ }^{4}$ Livret à la Bibliothèque de l'Opéra de Paris.

${ }^{5}$ Sous le titre Le démon d'Anjou.

${ }^{6} 2^{e}$ partie, chap. XXXI.
} 
la razo; Bertran perd Hautefort, - «là-haut sur le rocher abrupt/ fument les ruines de Hautefort»-mais en quatre strophes, il le regagne grâce à la puissance de ses chants, chacune évoquant un poème fameux, de l'appel aux conjurés Ventadorn et Comborn, au planh pour Henri le Jeune. Devant cette figure emblématique s'incline le souverain. De même, $H$. Heine, en 1841, compose une romance sur « Bertrand le Troubadour », nouvel Orphée charmant les grands fauves royaux. Par ces deux poèmes courts et frappants, Bertran passe dans les manuels scolaires; l'atlas historique de Putzgers, paru à Leipzig en 1916, indique, à côté de Muret et Chalus, lieux où moururent des rois, à la charnière du XII ${ }^{e}$ et du XIII siècles, «Autafort » selon la graphie de la ballade de Uhland.

En Allemagne s'entrelacent donc la recherche philologique, les cours des romanistes et la réflexion sur les rapports entre indépendance nationale et création poétique. Ces diverses perspectives entrainnent aussi bien la connaissance précise des manuscrits et la première édition critique, celle de Stimming en 1879, que la célébration du troubadour aquitain par des récritures de sirventes aux Jeux Floraux de Cologne en 1902; ou encore la reprise des traductions de Diez par un professeur de composition au conservatoire de Dresde, grand admirateur de Liszt, Felix Dräseke. Tandis que Moyen Age, opéras ou grands drames lyriques wagnériens vont de pair, il écrit livret et musique pour un Merlin puis, en 1894, l'opéra Bertran de Born, qui ne sera jamais porté à la scène' .

Suivre les réincarnations du chevalier-poète nous renseigne sur l'importance pour tout le nord de l'Europe au moins, des Universités allemandes, qui rayonnent sur Copenhague ou sur les institutions académiques russes. En 1872, un jeune Danois qui termine des études de philosophie et sera connu comme un prolixe poète de circonstances, traducteur de La pucelle d'ortéans de Schiller, des œuvres de Rostand et de La fille de Roland de H. Bomier, Ernst von Recke, se lance dans un premier drame lyrique de 175 pages. Plus étonnant, on écoutera les échos des sirventes dans cette «Rossia » citée par le troubadour Peire Bremon Ricas Novas comme l'espace du lointain, pour ne pas dire de la barbarie. Et ce en pleine période révolutionnaire! En 1923, parait dans la revue littéraire et artistique de Petersbourg La Ville, une courte tragédie, Bertran de Borm, jamais mise en scène, mais appréciée par Gorki. De fait, un an plus tard son jeune auteur Lev Lunts, étudiant brillant du Gymnase de Petersbourg où il se distingua dans l'étude des langues et littératures romanes, est emporté par la maladie. Figure de proue d'un des groupes littéraires les plus en vue, «les Frères de Sérapion» rassemblés autour de Zamiatine, ni futuristes, ni prolétariens, il est l'auteur d'un manifeste Vers l'Occident qui vise à renouveler par la maîtrise de la culture occidentale l'inspiration russe.

Pour ces trois hommes de thÊâtre, l'élargissement du champ spatial de leurs intérêts correspond à l'ouverture du temps des royaumes, temps des «nations", des voyages des étudiants et des moines, temps des alliances. Ce temps d'avant les frontières étatiques donne aisément naissance à une géographie européenne. Dans le drame danois, les scènes font défiler l'Angleterre avec la cathédrale de Canterbury et le tombeau de Thomas Becket, les châteaux de Normandie, tout en évoquant, par le retour de Croisade, la Terre Sainte. Pour Dräseke, il s'agit de faire imaginer un vaste espace, aimanté par Hautefort: dans les deux premiers actes, Rouen et la Normandie des Plantagenêts voient l'arrivée d'envoyés de

\footnotetext{
${ }^{7}$ Livret à la Bibliothèque de Dresde.
} 
Bohême et un départ à la Croisade. L'opposition entre le nord et le sud très prisée par les Romantiques, Augustin Thierry en tête, mais aussi par le Genevois Sismondi, laisse la place à une séparation est/ouest, entre le domaine des Barbares et la douce France.

En effet, l'image méridionale se résout en quelques stéréotypes: «accent charmant du pays d'oc » pour l'écrivain danois, clichés colorés des danseurs en costumes espagnols et de la belle gitane (sic) pour Dräseke. On ne trouvera ni le personnage d'Alphonse d'Aragon ni celui de Raymond V de Toulouse, l'ennemi et l'allié qui ouvrent le premier sirventes attesté de Bertran Lo coms m'a mandat e mogut, alors que le roi d'Aragon est aussi au centre de deux des plus beaux sirventes guerriers puis de la plus célèbre razo en prose. Chez Lunts, vivre presque en prisonnier à Argentan et Clairvaux aiguise les regrets pour un trop lointain et déjà perdu Hautefort. Ce qui se construit dans ces textes c'est au coeur de l'Europe du Moyen Age, moins l'Occitanie que la France, la France des bords de Seine, la France de saint Bernard de Clairvaux, la douce France de Roland solitaire, référence pour l'écrivain danois. Pourtant si l'on avait dû en croire Bertran de Born, la France était alors en proie à la décadence du fait de la prudence mesquine et de l'avarice de son roi Philippe Auguste.

\section{Un Moyen Age d'enluminure pour un théâtre de l'énergie}

Alors que la dramatisation dialoguée caractérise déjà le choix des poètes ainsi que le découpage en feuilleton du roman de Mary-Lafon, paru en 1839 mais repris dans l'hebdomadaire Le Joumal du Dimanche en 1865, les réincarnations de Bertran de Bom se font en majorité au théâtre; on verra aussi en France, en 1936, un Bertran de Born, celui de Valmy-Baysse, joué par un sociétaire de la Comédie Française, devant le mur du thêâtre d'Orange, avec une musique de Darius Milhaud.

Le Moyen Age inspire d'abord des images: champ de bataille et les tentes si fréquentes dans les enluminures, remparts et silhouette de donjon. Se dessine sur la scène un univers de monuments chargés de sens dans leur stylisation, sous le double signe de la guerre et de Dieu: ombre du monastère dans l'opéra italien, tombeau de martyr dans la cathédrale de Canterbury et croisade évoquée de Poncchielli à Drasëke beaucoup plus que dans les textes de Bertran de Born, ou que dans les visions romantiques des chantres de l'Occitanie tel Mary-Lafon. Poncchielli, Recke, Drasëke jouent des contrastes entre l'incendie, le feu des cierges et les ombres encapuchonnées, dans une ambiance souvent nocturne chère aux ballades allemandes. Murs et donjons tombent avec toute la violence des époques primitives tandis que la terre, le feu et l'eau retrouvent leur force cosmique.

Le drame lyrique correspond particulièrement bien à ce que le public imagine du Moyen Age et de ses créations poétiques; sauvagerie dans le feu et les cuivres, naïveté des chansons paysannes. Abondent les motifs musicaux notés comme médiévaux. Les fanfares accompagnent le passage des rois et les assauts des guerriers tandis que leur fait contrepoint la musique religieuse, chants de pèlerin, chansons de croisade ou litanies funèbres. L'œuvre danoise jouée une trentaine de fois au Théâtre Royal de Copenhague en 1872 sera ainsi reprise sous forme de drame lyrique en 1908. Seul Lunts échappe à ces clichés dans ses cinquante pages emportées par un tempo rapide.

Pour le XIX siècle des keepsakes et des romans historiques, sinon pour Bertran de Bom, auteur de peu de cansos, qui dit troubadour dit Amour. Cette thématique sert pourtant chez 
nos auteurs plutôt de simple prétexte à l'intrigue dramatique. Il leur faut recourir à des inventions diverses jouant sur des émotions fortes qui n'ont rien à voir avec la casuistique du fin amor. A son raffinement, difficile à saisir, on préférera le tragique du mariage imposé ou refusé à des personnages féminins, souvent fragiles, dominés par un père, un roi, ou une promesse. Bertran s'affirme comme un être de passion exclusive: négative dans l'opéra italien, exaltante pour le drame danois, destructrice chez Drasëke. Lunts, moins tenté par le mélodrame, insiste sur la séduction facile et les infidélités. Aucun ne se fonde ni sur la thématique de l'amour de loin ni sur l'allégresse du jeu poétique de la "dame empruntée », miroir multiple de toutes les beautés féminines, non plus que sur l'ironie sensuelle propre au troubadour de Hautefort.

L'héroïsme se lira ailleurs que dans la thématique amoureuse. Même si nous laissons de côté la légende noire, sortie de Dante, de l'opéra italien et ses fureurs de mélodrame, partout surgit l'homme des affrontements avec Henri II. Rebelle car épris de justice (Recke), ou rebelle car passionné d'indépendance (Drasëke, Lunts). Le premier n'affronte pas un souverain-suzerain à qui tout vassal doit obéissance mais un tyran éclaboussé par le sang de Thomas Becket. Selon les autres, ni le gain, ni l'autorité ni même la prudence ne peuvent freiner les insultes et l'ironie de Bertran. Notre héros est un individu qui revendique sa liberté et la fait revendiquer aux autres: chez Drasëke, la fille du roi d'Angleterre n'acceptera plus un mariage politique et s'enfuira avec lui. Libre d'aimer, de combattre, de risquer sa tête, le chevalier médiéval? Comme chez les poètes romantiques Uhland et Heine, il s'agit d'une exaltation de la Liberté dans l'exception et la solitude pour un chevalier sans affins, sans alliés, sans les solidarités multiples qui font la société féodale. Ignorance des réalités historiques? Préférence romantique? Lecture sans recul des sirventes? Les lecteurs du troubadour sont tombés dans le piège des poèmes nés de la crise de la chevalerie menacée, dès la génération de Bertran de Bom, par la concurrence des routiers et mercenaires; «l'aristocratie s'abrite derrière un mythe celui d'une guerre courageuse, une guerre où l'individu, contrairement à la réalité, joue un rôle décisif ${ }^{8}$. Loin d'être un stratège qui regrouperait des alliés autour d'une cause commune, le Bertran de Born de la scène essaie de convaincre d'autres individus, barons, chevaliers, princes et princesse de faire usage de leur liberté et, pour ce faire, n'hésite ni devant la ruse ni devant la traitrise.

L'exaltation de l'indépendance comme valeur suprême, destructrice des normes morales mais non sans grandeur et beauté, déjà en germe dans l'opéra allemand, trouve un terrain favorable sur fond de tourmente révolutionnaire, celle que vit Lunts. Le Périgord a brûlé, Hautefort est détruit. Une fois abandonnés château et libertés, le vicomte à la cote trouée est "un dépenaillé, un turbulent, un insolent", fourbe s'il le faut; "une bête sauvage», s'inquiète le Jeune Roi. Pour la Liberté, c'est à dire pour reprendre sa vie à Hautefort, il a déjà donné Amour et Honneur. «Maintenant que meure aussi l'Amitié! ». L'auteur éclaire ses intentions dans la postface: «J'ai vécu dans la Révolution et je n'aurais pu écrire cette pièce sans la Révolution, c'est la Tragédie de la Révolution et de la Liberté, celle du bourgeois russe à qui l'on a pris ses meubles ». Tragédie de l'homme qui se bat contre le pouvoir étatique. Le poète à qui l'on a pris son château de Hautefort et enlevé la possibilité

\footnotetext{
${ }^{8}$ G. Duby, «L'Europe féodale : l'ordonnancement de la paix », Concetti, storia, miti e immagini del medio Evo, Florence, 1973, p. 482.
} 
de blâmer, celui qui refuse la prison dorée du poète de cour, tente vainement de faire obstacle à un processus d'évolution historique que Lunts vit au quotidien, la formation sanglante d'un Etat. Zamiatine, le maitre des «frères de Sérapion » écrivait: «il n'est de vraie littérature que produite non par des fonctionnaires bien-pensants et zélés, mais par des fous, des ermites, des hérétiques, des rêveurs, des rebelles et des sceptiques», Bertran de Born serait l'un d'eux.

Un poète donc. L'actualisation ou la nostalgie se jouent dans le contact avec les vers de ce poète. Pour Recke, plus fantaisiste et prolixe, on ne notera que le plus célèbre sirventes guerrier, Bem platz, à côté d'inventions comme le chant de Roland ou un conte arabe interprétés par Bertran, déguisé en ménestrel. Huit poèmes ainsi enchâssés, ainsi que le prologue où dans la clarté des bougies bruissent cristaux et soieries, rappellent les clichés des « almanachs du troubadour ». Drasëke fait entendre le chant du jongleur Papiol : sirventes sur les plaisirs de la guerre, canso, Ges de disnar, planh pour le jeune roi et ballades de Uhland et Heine pour le dénouement. Hautefort s'écroule, les femmes aimées meurent mais la voix du poète résiste. Quant à Lunts, qui reconnaissait l'anachronisme de sa vision, il la défendait au nom de l'admiration pour un poète de l'ardeur. Il cherchait, grâce à ce personnage, à installer dans le nouveau théâtre russe un ton qui se distinguât des "ruminations psychologiques d'un Tchekhov », à créer un rythme plus vif. «La main meurt tandis que les mots survivent ", aussi intègre-t-il l'aube de Giraut de Bornelh et l'escondig de Bertran. II abandonne le vers tragique qu'il considère trop littéraire comme la prose trop teme, en alternant les vers libres, une prose elliptique et quelques anapestes, pour donner l'impression d'un rythme précipité, parfois heurté, où les pauses poétiques ne durent que l'espace d'un rêve.

Tandis que le troubadour guerrier inspire les poètes en Europe, chacun selon les styles en vogue, en France, il dévoile surtout les politiques. Militant de causes parfois inattendues (légitimiste ou ancien combattant "haïsant le Saxon roux"), il y assume la fonction explicative et mobilisatrice des mythes politiques. Selon les circonstances historiques nationales, il sera comparé à Vercingétorix. Du Guesclin ou Jeanne d'Arc. Les œuvres thêâtrales représentées en 1925 à Monte Carlo ${ }^{9}$, et en 1936 à Orange, évitent les reprises des strophes les plus violentes où la destruction est exaltée comme le plaisir suprême, ce qui vise à souligner combien le héros, plus chevaleresque que guerrier, se sacrifie à de justes combats. Mais il ne passe pas dans la culture générale. L'Histoire de France de Lavisse n'y fera qu'une brève allusion, pour lui dénier toute importance. Bertran de Born ne figure pas un héros susceptible d'être "républicanisé "; la Troisième République n'apprécia ni les rebelles féodaux ni les patriotismes autres que français.

En matière d'exemplarité, sur la scène européenne du siècle où l'on réintègre vraiment la culture occitane au panorama historique général et aux connaissances linguistiques et littéraires, ces œuvres nous semblent représentatives de la connaissance moyenne des publics cultivés. Bertran de Bom retient l'attention comme individu libre plus que comme symbole de l'honneur ou de la chevalerie, sans attaches autres que celles qu'il se donne et par làmême moins occitan que prévu. Il est vrai que le Midi, ou la Provence, de la fin du siècle

${ }^{9}$ R. Charbonnel, Bertran de Bom, Fayard, Paris, 1930. 


\section{Martine DAUZIER}

s'inscrivait un peu partout, et jusque chez Mistral, comme le lieu d'une allègre et exotique frivolitét ${ }^{10}$.

Bertran de Bom guerroie pour toujours à la face des princes au carrefour de l'Histoire, une histoire incertaine, et de la Poésie, une poésie plus reconnue qu'on ne l'aurait cru au nord de l'Europe. Il s'agit d'une floraison de rêves individuels et de modes dans des œuvres parfois de second ordre mais qui participent d'une grande communauté de passions et de valeurs pour forger une image du Moyen Age européen. Chez tous, il demeure l'un des troubadours. Nous voyons ici que, comme le dit J. Roubaud,

«le spectre des troubadours hante la poésie. (...)Or la poésie du trobar, dans cette langue au futur précaire, langue sans nation, langue sans postérité ininterrompue malgné les éclats baroques et les reconnaissances volontaires depuis Mistral, est la première poésie moderne en Europe après la chute de l'empire romain (...) La poésie, si elle est un art formel de la langue, une mémoire de la langue, ne peut complètement oublier le trobar ${ }^{11} "$.

Pour autant, survivent les poètes aimés par d'autres poètes. "Gerbe de miroirs brisés" disait des poèmes de Bertran de Born, qu'il fit revivre dans les siens, E. Pound - que, au nom des langues entrelacées de son poème Near Perigord de 1915, nous aurions pu annexer à nos itinéraires européens, anglais, français et provençal -, gerbe toujours à lier.

Martine Dauzier

(Université de Paris XII)

\footnotetext{
${ }^{10}$ Voir Ph. Martel, «Les historiens du début du XIX siècle et le Moyen Age occitan : Midi éclairé, Midi martyr ou Midi pittoresque ", Romantisme, 35, 1982, p. 49 sq.

${ }^{11}$ J.Roubaud, La fleur inverse, Paris, 1986, pp. 345-345.
} 